\title{
TRIM5 $\alpha$ and TRIMCyp form apparent hexamers and their multimeric state is not affected by exposure to restriction-sensitive viruses or by treatment with pharmacological inhibitors Marie-Édith Nepveu-Traversy, Julie Bérubé and Lionel Berthoux*
}

Address: Laboratory of retrovirology, University of Québec, Trois-Rivières, QC, G9A 5H7, Canada

Email: Marie-Édith Nepveu-Traversy - nepveutr@uqtr.ca; Julie Bérubé - julie.berube1@uqtr.ca; Lionel Berthoux* - lionel_berthoux@yahoo.com

* Corresponding author

Published: 3 November 2009

Retrovirology 2009, 6:100 doi:10.1186/1742-4690-6-100

This article is available from: http://www.retrovirology.com/content/6/1/100

(c) 2009 Nepveu-Traversy et al; licensee BioMed Central Ltd.

This is an Open Access article distributed under the terms of the Creative Commons Attribution License (http://creativecommons.org/licenses/by/2.0), which permits unrestricted use, distribution, and reproduction in any medium, provided the original work is properly cited.

\begin{abstract}
Proteins of the TRIM5 family, such as TRIM5 $\alpha$ and the related TRIMCyp, are cytoplasmic factors that can inhibit incoming retroviruses. This type of restriction requires a direct interaction between TRIM5 proteins and capsid proteins that are part of mature, intact retroviral cores. In such cores, capsids are arranged as hexameric units. Multiple lines of evidence imply that TRIM5 proteins themselves interact with retroviral cores as multimers. Accordingly, stabilization by crosslinking agents has revealed that TRIM5 $\alpha$ and TRIMCyP are present as trimers in mammalian cells. We report here that TRIM5 proteins seem to form dimers, trimers, hexamers and multimers of higher complexity in mammalian cells. The hexameric form in particular seems to be the most abundant multimer. Multimerization did not involve disulfide bridges and was not affected by infection with restriction-sensitive viruses or by treatment with the known TRIM5 inhibitors arsenic trioxide, MGI32 and cyclosporine A. We conclude that TRIM5 multimerization results from more than one protein-protein interface and that it is seemingly not triggered by contact with retroviral cores.
\end{abstract}

\section{Findings}

TRIM proteins form a family with dozens of members, most of them bearing a tripartite motif composed of a RING, B-box and Coiled-coil domains [1]. Restriction of retroviruses by members of the TRIM5 subfamily of TRIM proteins, which comprises the primate proteins TRIM $5 \alpha$ and TRIMCyp [2-4], is initiated by physical recognition of the incoming retrovirus by TRIM 5 proteins. This interaction occurs within the first hours following virus entry [5] and involves determinants present in the $\mathrm{N}$-terminal domain of the capsid proteins which constitute the retroviral outer core structure [6-8]. Retroviral capsid cores are assembled from hundreds of capsid proteins and the basic capsomer is a hexamer [9-11]. Restriction necessitates capsid proteins of the incoming retrovirus to be correctly mat- urated by the retroviral protease $[12,13]$. This is a required step for the core to adopt its final structure. In addition, mutations that affect the stability of the retroviral core interfere with the efficiency of restriction $[12,13]$. Virusfree capsid proteins, which do not multimerize to form cores, do not interact with TRIM5 proteins in cells [14]. That TRIM5-mediated restriction requires assembled retroviral cores brings the question of whether TRIM5 proteins themselves must be present as multimers. TRIM proteins are known to homomultimerize through their coiled-coil domain [1], which is required for restriction [15]. TRIM5 proteins from different species can interact with each other and in doing so can interfere with each other's restriction activity [16]. TRIM5 $\alpha$ has also been shown to trap incoming retroviral particles inside cyto- 
plasmic bodies, which further suggests that TRIM5 proteins interact with their targets as multimers [17]. TRIM5 $\alpha$ and TRIMCyp have been stabilized as trimers by treatment with cross-linking agents [18-23]. Some undefined higher-order multimers have been occasionally observed $[18,19]$. The relevance of trimerization was confirmed by the fact that modified TRIMCyp, in which the coiled-coil domain is substituted by that of a trimeric heterologous protein, restricted HIV-1, although at much lower levels than wild-type TRIMCyp did [19]. A recombinant TRIM5 protein expressed in insect cells was observed as dimers [21] and minor amounts of dimeric TRIM5 $\alpha$ have been observed in cells [23]. However, dimerization/trimerization of TRIM5 proteins fails to explain the formation of cytoplasmic bodies or the sequestration of incoming restricted virus in such structures. Thus, we analyzed TRIM $5 \alpha /$ TRIMCyp multimerization in the presence or absence of restriction-sensitive viruses and upon treatment with various drugs that inhibit the restriction process.

We first analyzed TRIM5 multimerization in stably transduced Mus dunni tail fibroblast (MDTF) cell lines [24]. Multimers were stabilized by treatment with glutaraldehyde as first described by Mische and collaborators [23]. Surprisingly, TRIM $5 \alpha_{\mathrm{rh}}$ was not present as a trimer in these cells. Rather, we observed a band with a size in the 300$400 \mathrm{kDa}$ range (Fig. 1), and subsequent experiments that used a different molecular weight marker confirmed this apparent weight. Since the TRIM $5 \alpha_{\mathrm{rh}}$ monomer migrates at 55 to $60 \mathrm{kDa}$, this multimer may be a hexamer. Higherorder multimers were also seen but their size could not be estimated. These high molecular weight multimers were present in the stacking gel when they were seen; and in some experiments they were found to have barely penetrated the acrylamide. We cannot exclude that they might be aggregates rather than genuine higher-order assemblies of TRIM5 $\alpha$. TRIMCyp was found in MDTF cells as dimers and trimers and also as higher-order multimers that included a band slightly heavier than the $250 \mathrm{kDa}$ marker (Fig. 1). Since monomeric TRIMCyp migrates at about 45 $\mathrm{kDa}$, the multimer seen is most likely a hexamer (although the migration pattern of multimeric complexes might be different from those of linear proteins). Higher amounts of glutaraldehyde were required to reveal the presence of hexamers and higher-order multimers, compared with dimers or trimers. Thus, TRIM5 $\alpha$ and TRIMCyp can have distinct multimerization profiles despite both being fully active in MDTF cells. They also share the capacity to form apparent hexamers. Because coiled-coil domains can dimerize through the formation of covalent disulfide bridges between cysteine residues in some instances [25], we performed a Western blot analysis of TRIM5 $\alpha$ and TRIMCyp in reducing and nonreducing conditions. In the absence of $\beta$-mercaptoethanol, both
TRIM5 $\alpha$ and TRIMCyp were less easily detected, but migrated at the expected size; and no dimer or more complex multimers were visible (Fig. 1B), with the exception of very high molecular weight structures which seemed to be present in higher amounts compared to the reducing conditions. Thus, it appears that disulfide bridges do not induce TRIM5 protein dimers, trimers or hexamers, but perhaps they are involved in the formation of non-specific aggregates.

To investigate the possibility that TRIMCyp multimerization was induced or modulated by exposure to a restriction-sensitive virus, we repeated the glutaraldehyde crosslinking assay after 6 hours of continuous infection with TRIP-CMV-GFP, which is an HIV-1 vector encoding GFP $[24,26]$. Approximately $1 \%$ of the MDTF-TRIMCyp cells were infected in these conditions, versus more than $50 \%$ of the same cells not expressing TRIMCyp (not shown). Thus, TRIMCyp restriction activity was not saturated at this multiplicity of infection (MOI), yet cells were exposed to large amounts of HIV-1 virions in order to maximize the frequency of TRIMCyp:capsid interaction. However, HIV-1 infection did not noticeably modify the relative amounts of TRIMCyp trimers, hexamers and higher-order multimers (Fig. 2A). We repeated the experiment in the presence of cyclosporine A (CsA), which completely abrogates the restriction mediated by TRIMCyp as it binds to the same CypA domain that recognizes HIV-1 capsid proteins $[27,28]$. CsA treatment, however, had no effect on TRIMCyp multimerization profiles, further implying that multimerization was independent of specific virus recognition.

It was recently reported that TRIM5 $\alpha$ and TRIMCyp are degraded in a proteasome-dependent pathway following infection with a restriction-sensitive retrovirus [29]. Thus, it was conceivable that in our previous experiment HIV-1 modulated the multimerization of only a part of the cellular TRIMCyp proteins which were then degraded by the proteasome. To address that possibility, we repeated the experiment in the presence of the proteasomal inhibitor MG132, thereby preventing virus-induced TRIMCyp targeting to the proteasome (not shown). In addition we infected with a higher dose of the HIV-1 vector, leading to $20 \%$ infected cells. TRIMCyp restriction activity was significantly saturated at this MOI, implying that most TRIMCyp proteins that were restriction-competent at the time of infection were indeed engaging incoming HIV-1 [24]. However, MG132 did not appreciably modify the multimerization profile of TRIMCyp in the absence or presence of HIV-1 (Fig. 2B). Like before, dimers, trimers and higher-order multimers were formed. The band corresponding to putative hexamers was less well-defined compared with previous experiments, but this is probably due 
$\underline{\mathbf{A}}$
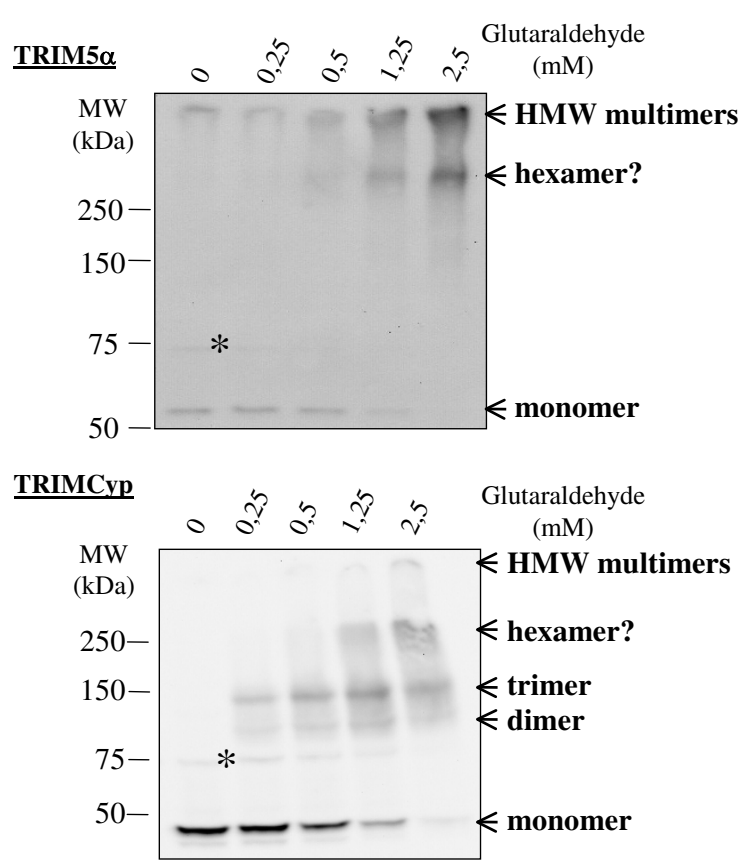

$\underline{\mathbf{B}}$

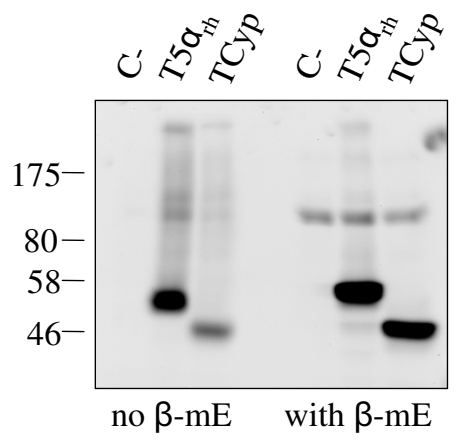

Figure I

Multimerization profiles of TRIM5 $\alpha$ and TRIMCyp. A, $0.5 \%$ NP40 lysates were prepared from Mus dunni tail fibroblast cells (MDTFs) stably expressing FLAG-tagged TRIM $5 \alpha_{r h}$ or owl monkey TRIMCyp. The soluble fraction of each lysate was divided in aliquots that were treated for $5 \mathrm{~min}$ with the indicated glutaraldehyde concentrations before proteins were denatured by boiling in the presence of SDS. Proteins were then separated on an $8 \%$ polyacrylamide gel, transferred to a nitrocellulose membrane, and probed with a rabbit anti-FLAG antibody (Cell Signaling). The apparent multimeric states are indicated on the right as deduced from the size of the bands. The star indicates an unspecific protein cross-detected by the FLAG antibody. B, Lysates were prepared from HeLa cells stably transduced with the same constructs as above and in the absence or presence of $100 \mu \mathrm{M}$ $\beta$-mercaptoethanol as indicated. to technical reasons unrelated to the effects of MG132 on TRIM5.

We used MDTF cells expressing TRIM5 $\alpha$ cloned from Vero cells (African green monkey) [24] to investigate whether, unlike that of TRIMCyp, TRIM5 $\alpha$ multimerization could be modulated upon infection with a restricted virus. This orthologue of TRIM5 $\alpha$ decreases HIV-1 replication by about 100-fold [30] and also inhibits the N-tropic strains of the murine leukemia virus (MLV), although to a smaller extent (10-fold or less) [24]. As in Fig. 2, we challenged these cells with restricted (HIV-1 and N-MLV) or non-restricted (B-MLV) viruses at relatively high doses and in presence of MG132 (Fig. 3). Under these conditions, the inhibition of N-MLV by TRIM $5 \alpha_{A G M}$ was lower than previously observed, a likely consequence of the MG132 treatment and of the high MOI (not shown). TRIM $5 \alpha_{\text {AGM }}$ formed apparent trimers and hexamers in these cells but no dimers were observed (Fig. 3A), nor did we see multimers of very high molecular weight in this particular experiment. Challenges with the different viruses had little effect on the multimerization pattern. The relative number of hexamers stabilized at the highest glutaraldehyde concentration used, decreased slightly in cells infected with one of the restricted viruses (HIV-1; Fig. $3 \mathrm{~A})$, but increased slightly in cells infected with the other restricted virus (N-MLV; Fig. 3B). No notable differences were found at the other glutaraldehyde concentrations. Data from Fig. 2 and 3 together suggest that the multimerization of TRIM5 proteins is not modulated by retroviral infections. A caveat in these experiments, however, is that the percentage of TRIM5 proteins actually engaged in the restriction process at any given time is not known. Even at high multiplicities of infection, it is still possible that modulation of multimerization occurs at levels undetectable in our assays.

Arsenic trioxide $\left(\mathrm{As}_{2} \mathrm{O}_{3}\right)$ inhibits the restriction activity of TRIM5 proteins in a virus-independent, TRIM5 orthologue-independent, cell type-dependent manner [30,31]. The mechanism of action of this drug on TRIM5 proteins is at present unknown. Thus, it was of interest to analyze whether it could affect the capacity of TRIM5 proteins to multimerize. We found that $\mathrm{As}_{2} \mathrm{O}_{3}$ did not affect TRIM5mediated restriction in MDTF cells (not shown), and thus we used human HeLa cells for this particular experiment. As expected, stable expression of TRIM $5 \alpha_{\mathrm{rh}}$ and of TRIMCyp in HeLa cells resulted in an approximately 100-fold reduction in permissiveness to transduction with an HIV1 virus expressing GFP (not shown). We found that both restriction activities were partly suppressed by $\mathrm{As}_{2} \mathrm{O}_{3}$ treatment (Fig. 4A). More precisely, a short (10 hours) treatment with $\mathrm{As}_{2} \mathrm{O}_{3}$ at the time of infection increased permissiveness to HIV-1 by up to 15 -fold in cells expressing TRIM $5 \alpha_{\mathrm{rh}}$ and 20-fold in cells expressing TRIMCyp, 

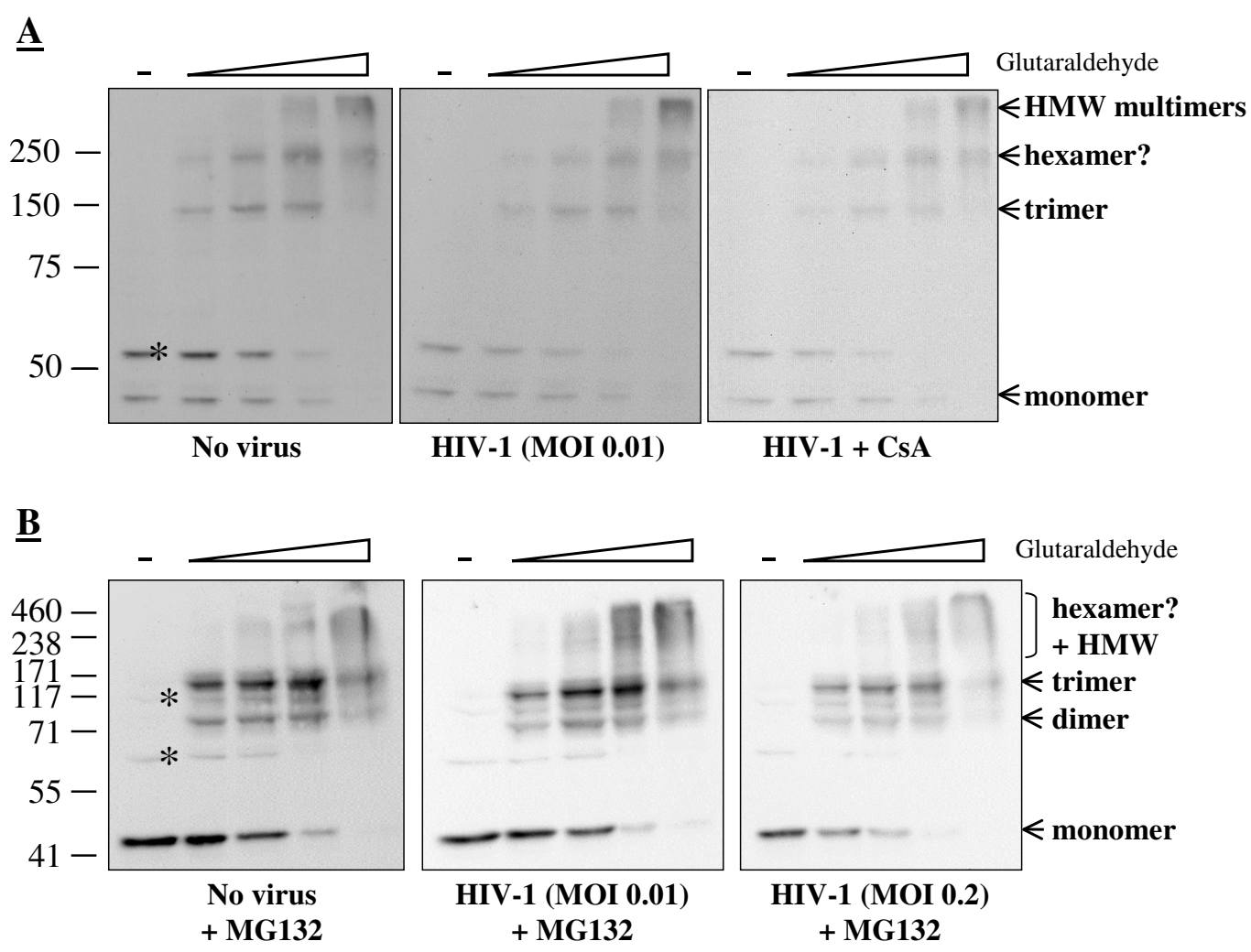

\section{Figure 2}

Multimerization of TRIMCyp in cells infected by HIV-I. A, MDTF-TRIMCyp cells were challenged with an HIV-I vector expressing GFP, at a dose leading to infection of about $1 \%$ of the cells and either in the presence or not in the presence of 5 $\mu \mathrm{M}$ cyclosporine A (Sigma). After 6 hours of infection, cells were lysed in presence of increasing glutaraldehyde concentrations as in Fig. I. Western blot analysis of FLAG-tagged proteins was performed as above. B, the experiment was repeated in the presence of I $\mu \mathrm{M} \mathrm{MGI} 32$ (Sigma) and using two different virus doses. The stars indicate cellular proteins cross-detected by the FLAG antibody as evidenced by analysis of lysates from parental cells (not shown).

while having a smaller, "background" effect of about 4fold in the control untransduced cells. Crosslinking assays yielded slightly different results in HeLa cells compared with what had been observed in MDTF cells. TRIM $5 \alpha_{\mathrm{rh}}$ did not dimerize but trimers were visible, as well as apparent hexamers and higher-order multimers (Fig. 4B, upper panel). TRIMCyp was found as dimers, trimers, and hexamers (Fig. 4B, lower panel). An additional band migrating faster than the hexamer was visible and could be a pentamer. In both cases, the experiment was done in the absence or presence of $\mathrm{As}_{2} \mathrm{O}_{3}$; and no differences were observed. Therefore, arsenic trioxide does not influence the multimeric state of TRIM $5 \alpha$ and TRIMCyp.

We find that in addition to the dimeric and trimeric forms previously described, TRIM5 $\alpha$ and TRIMCyp can form apparent hexamers and more complex multimers. Why discrete hexamers were not previously seen by others is probably only related to the difficulty of resolving high molecular weight complexes in acrylamide gels, although we cannot totally exclude that the C-terminus FLAG tag used in our constructs may somehow interfere with protein multimerization. Because of low expression levels in mammalian cells, it is not possible at this point to perform the biochemical experiments that would be needed to ascertain that the various multimers seen here are composed of TRIM5 proteins only. For instance, a trimer of TRIM5 could associate with a heterologous cellular protein, yielding a band resembling a TRIM5 hexamer. Thus, other approaches will be needed. The hexamer model is obviously appealing because capsid proteins are themselves organized as hexamers in mature retroviral cores. Thus, a hexamer of TRIM5 proteins could be needed to recognize a retroviral capsomer. Formation of dimers, trimers and hexamers, however, does not seem to be triggered by contact with a restricted retrovirus. It remains 
$\underline{\mathbf{A}}$

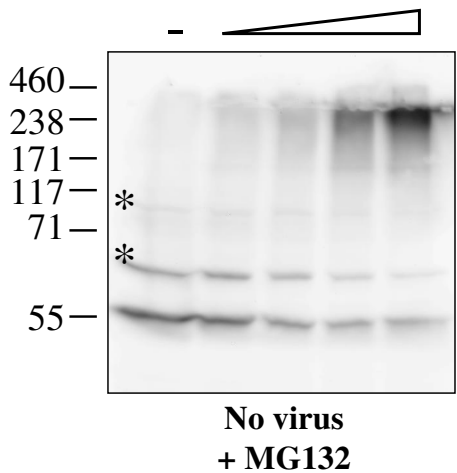

B

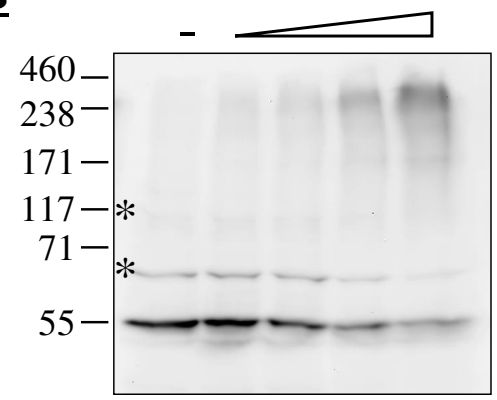

B-MLV (MOI 0.3)

+ MG132
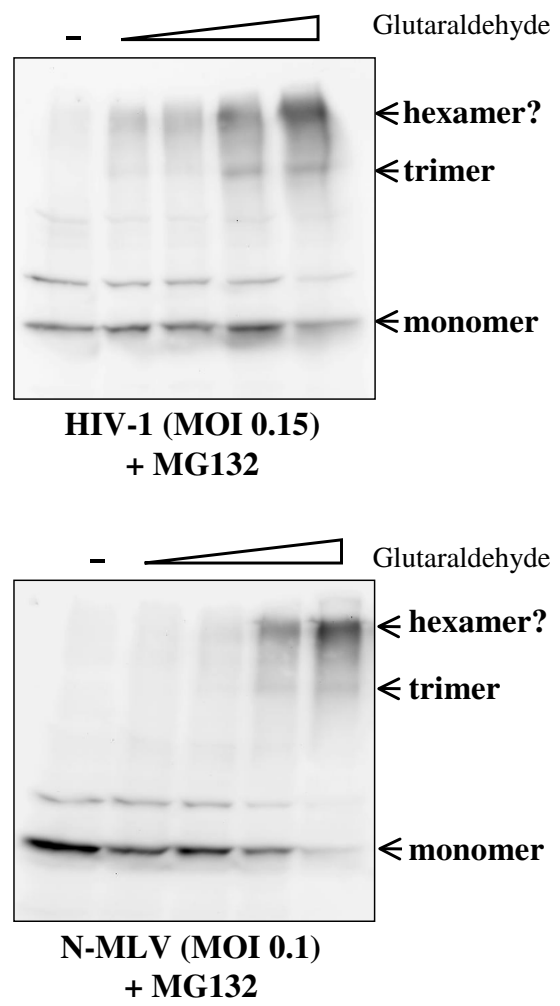

\section{Figure 3}

Multimerization of TRIM5 $\alpha_{A G M}$ is not modulated by infection with restriction-sensitive viruses. A, MDTF cells expressing TRIM $5 \alpha_{\text {AGM }}$ were either infected or not infected with HIV-I for 6 hours, using a virus dose leading to about I5\% infected cells and in the presence of I $\mu$ M MGI32. Crosslinking assays were done as before. B, MDTF-TRIM5 $\alpha_{A G M}$ cells were infected with identical amounts (as normalized by titration on parental cells) of B-MLV or N-MLV-derived vectors expressing GFP in the presence of MGI32. 30\% and 10\% of the cells were infected (GFP-positive), respectively, by B-MLV-GFP and NMLV-GFP, as seen by flow cytometry 2 days later.

possible that the nature and number of some specific higher order multimers not resolved in our gels could be modulated during the restriction process. Not surprisingly, the coiled-coil domain of TRIM5 proteins has been found to be required for the formation of trimers $[18,23]$. However, this does not imply that a single protein:protein interface present in this domain is responsible for the various multimeric forms observed. Rather, it is more likely that one interface would lead to dimerization and another one to trimerization; together they would be responsible for hexamerization. Perhaps yet other determinants within TRIM5 $\alpha$ and TRIMCyp lead to the formation of very high molecular weight multimers. Consistent with the existence of more than one molecular site of TRIM5:TRIM5 interactions, Li and Sodroski have recently reported that point mutants in the B-Box domain show normal multimerization patterns in crosslinking assays while being less efficient at engaging in protein:protein interactions through co-immunoprecipitation assays [22]. Regardless of what the exact molecular mechanism of TRIM5 multimerization is, our data suggest that TRIM5 multimerization is complex but that formation of low molecular weight multimers is not influenced by contact with a restricted retrovirus. 

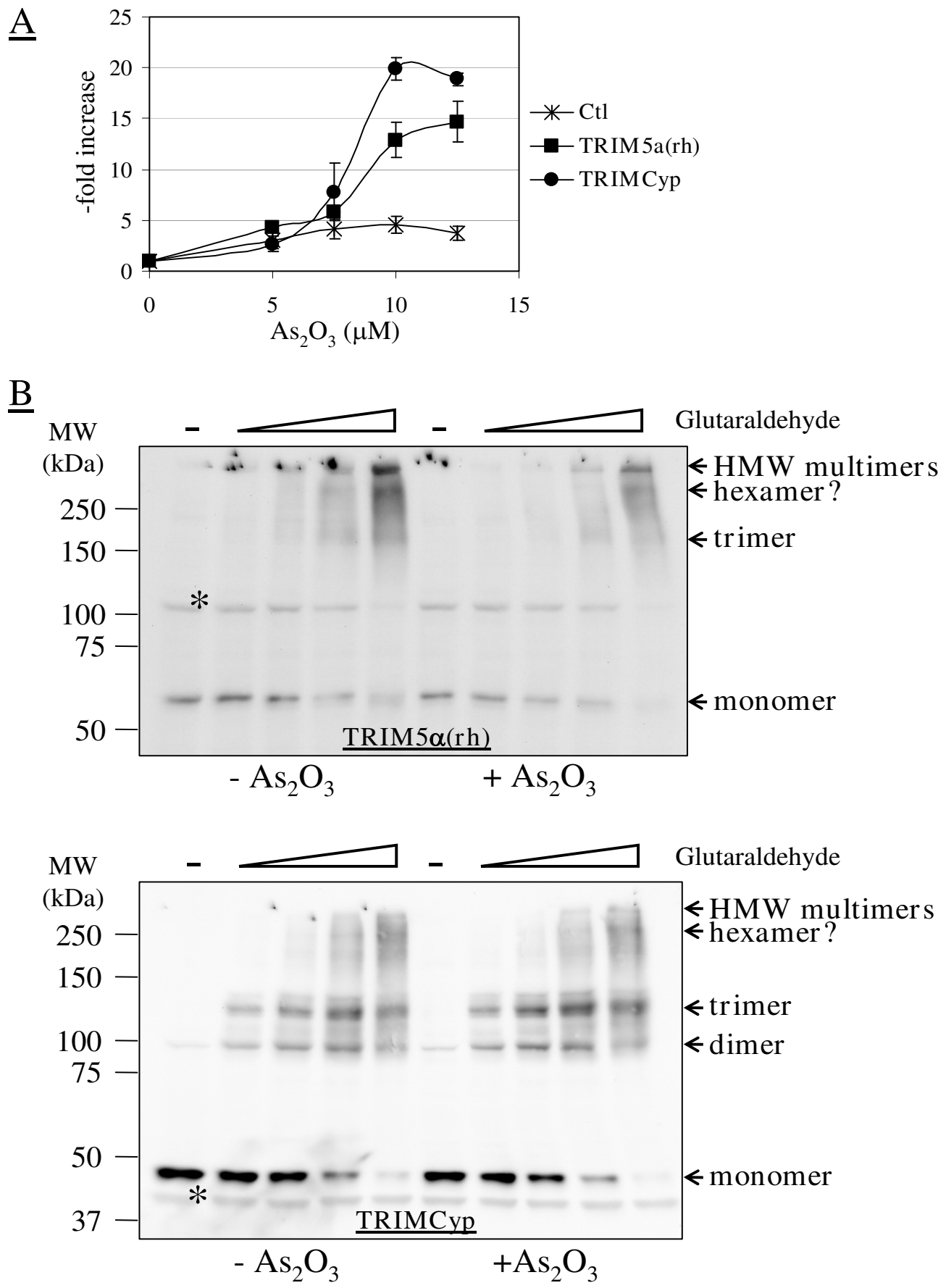

Figure 4

$\mathrm{As}_{2} \mathrm{O}_{3}$ does not modify the multimerization of TRIM5 $\alpha$ and TRIMCyp. A, human HeLa cells stably expressing TRIM5 $\alpha_{\mathrm{rh}}$ and TRIMCyp, or control untransduced cells, were challenged with an HIV-I vector expressing GFP at virus doses leading to about I\% infected (GFP-positive) cells in the absence of drug. Infections were performed in the presence of increasing $\mathrm{As}_{2} \mathrm{O}_{3}$ concentration (x-axis) and for 10 hours, after which supernatants were replaced with fresh medium to avoid $\mathrm{As}_{2} \mathrm{O}_{3}-$ related toxic effects. 2 days after infection, the \% of cells expressing GFP were determined by flow cytometry analysis. Results were expressed as -fold increase compared with the untreated control. The averages from triplicate infections with standard deviations are shown. B, glutaraldehyde assays were performed exactly as before and in the absence or presence of $10 \mu \mathrm{M}$ $\mathrm{As}_{2} \mathrm{O}_{3}$. 


\section{Competing interests}

The authors declare that they have no competing interests.

\section{Authors' contributions}

MÉNT, JB and LB designed the study. MÉNT and JB performed the experiments. LB drafted the manuscript. All authors read and approved the final draft.

\section{Acknowledgements}

We thank Mélodie B. Plourde for help with drafting the manuscript. This work was supported by the Canadian Institutes for Health Research and by the Canada Research Chairs program.

\section{References}

I. Reymond A, Meroni G, Fantozzi A, Merla G, Cairo S, Luzi L, Riganelli $D$, Zanaria E, Messali S, Cainarca S, et al.: The tripartite motif family identifies cell compartments. Embo / 200I, 20:2I40-2I5I.

2. Luban J: Cyclophilin A, TRIM5, and resistance to human immunodeficiency virus type I infection. J Virol 2007, 81:1054-1061.

3. Nisole S, Stoye JP, Saib A: TRIM family proteins: retroviral restriction and antiviral defence. Nat Rev Microbiol 2005, 3:799-808.

4. Towers GJ: The control of viral infection by tripartite motif proteins and cyclophilin A. Retrovirology 2007, 4:40.

5. Perez-Caballero D, Hatziioannou T, Zhang F, Cowan S, Bieniasz PD: Restriction of human immunodeficiency virus type I by TRIM-CypA occurs with rapid kinetics and independently of cytoplasmic bodies, ubiquitin, and proteasome activity. J Virol 2005, 79: I5567-15572.

6. Hatziioannou T, Cowan S, Von Schwedler UK, Sundquist WI, Bieniasz PD: Species-specific tropism determinants in the human immunodeficiency virus type I capsid. I Virol 2004, 78:6005-60I2

7. Ikeda Y, Ylinen LM, Kahar-Bador M, Towers GJ: Influence of gag on human immunodeficiency virus type I species-specific tropism. J Virol 2004, 78: I I816-I I822.

8. Owens CM, Song B, Perron MJ, Yang PC, Stremlau M, Sodroski J: Binding and susceptibility to postentry restriction factors in monkey cells are specified by distinct regions of the human immunodeficiency virus type I capsid. J Virol 2004, 78:5423-5437.

9. Li S, Hill CP, Sundquist WI, Finch JT: Image reconstructions of helical assemblies of the HIV-I CA protein. Nature 2000, 407:409-413.

10. Mortuza GB, Haire LF, Stevens A, Smerdon SJ, Stoye JP, Taylor IA: High-resolution structure of a retroviral capsid hexameric amino-terminal domain. Nature 2004, 43 I:48I-485.

11. Pornillos O, Ganser-Pornillos BK, Kelly BN, Hua Y, Whitby FG, Stout $C D$, Sundquist WI, Hill CP, Yeager M: X-ray structures of the hexameric building block of the HIV capsid. Cell 2009, 137:1282-1292.

12. Forshey BM, Shi J, Aiken C: Structural requirements for recognition of the human immunodeficiency virus type I core during host restriction in owl monkey cells. I Virol 2005, 79:869-875

13. Shi J, Aiken C: Saturation of TRIM5 alpha-mediated restriction of HIV-I infection depends on the stability of the incoming viral capsid. Virology 2006, 350:493-500.

14. Sebastian S, Luban J: TRIM5alpha selectively binds a restrictionsensitive retroviral capsid. Retrovirology 2005, 2:40

15. Perez-Caballero D, Hatziioannou T, Yang A, Cowan S, Bieniasz PD: Human tripartite motif 5 alpha domains responsible for retrovirus restriction activity and specificity. J Virol 2005, 79:8969-8978.

16. Berthoux L, Sebastian S, Sayah DM, Luban J: Disruption of human TRIM5alpha antiviral activity by nonhuman primate orthologues. J Virol 2005, 79:7883-7888.
17. Campbell EM, Perez O, Anderson JL, Hope TJ: Visualization of a proteasome-independent intermediate during restriction of HIV-I by rhesus TRIM5alpha. J Cell Biol 2008, I 80:549-56I.

18. Diaz-Griffero F, Vandegraaff N, Li Y, McGee-Estrada K, Stremlau M, Welikala S, Si Z, Engelman A, Sodroski J: Requirements for capsidbinding and an effector function in TRIMCyp-mediated restriction of HIV-I. Virology 2006, 35 I:404-4I9.

19. Javanbakht H, Diaz-Griffero F, Yuan W, Yeung DF, Li X, Song B, Sodroski J: The ability of multimerized cyclophilin $A$ to restrict retrovirus infection. Virology 2007, 367:19-29.

20. Javanbakht H, Yuan W, Yeung DF, Song B, Diaz-Griffero F, Li Y, Li X, Stremlau M, Sodroski J: Characterization of TRIM5alpha trimerization and its contribution to human immunodeficiency virus capsid binding. Virology 2006, 353:234-246.

21. Langelier CR, Sandrin V, Eckert DM, Christensen DE, Chandrasekaran V, Alam SL, Aiken C, Olsen JC, Kar AK, Sodroski JG, Sundquist WI: Biochemical characterization of a recombinant TRIM5alpha protein that restricts human immunodeficiency virus type I replication. J Virol 2008, 82: I | 682- I I694.

22. Li X, Sodroski J: The TRIM5alpha B-box 2 domain promotes cooperative binding to the retroviral capsid by mediating higher-order self-association. J Virol 2008, 82: I I 495-I I 502.

23. Mische CC, Javanbakht H, Song B, Diaz-Griffero F, Stremlau M, Strack $\mathrm{B}, \mathrm{Si}$ Z, Sodroski J: Retroviral restriction factor TRIM5alpha is a trimer. J Virol 2005, 79: I4446-I 4450.

24. Berube J, Bouchard A, Berthoux L: Both TRIM5alpha and TRIMCyp have only weak antiviral activity in canine DI7 cells. Retrovirology 2007, 4:68.

25. Walter JK, Rueckert C, Voss M, Mueller SL, Piontek J, Gast K, Blasig IE: The oligomerization of the coiled coil-domain of occludin is redox sensitive. Ann N Y Acad Sci 2009, I I 65: 19-27.

26. Zennou V, Petit C, Guetard D, Nerhbass U, Montagnier L, Charneau $P$ : HIV-I genome nuclear import is mediated by a central DNA flap. Cell 2000, I01:173-185.

27. Sayah DM, Sokolskaja E, Berthoux L, Luban J: Cyclophilin A retrotransposition into TRIM5 explains owl monkey resistance to HIV-I. Nature 2004, 430:569-573.

28. Towers G], Hatziioannou T, Cowan S, Goff SP, Luban J, Bieniasz PD: Cyclophilin A modulates the sensitivity of HIV-I to host restriction factors. Nat Med 2003, 9: I |38- I I 43.

29. Rold CJ, Aiken C: Proteasomal degradation of TRIM5alpha during retrovirus restriction. PLoS Pathog 2008, 4:e I000074.

30. Sebastian S, Sokolskaja E, Luban J: Arsenic counteracts human immunodeficiency virus type I restriction by various TRIM5 orthologues in a cell type-dependent manner. I Virol 2006, 80:205I-2054.

31. Berthoux L, Towers GJ, Gurer C, Salomoni P, Pandolfi PP, Luban J: As(2)O(3) enhances retroviral reverse transcription and counteracts RefI antiviral activity. J Virol 2003, 77:3167-3।80.

Publish with Bio Med Central and every scientist can read your work free of charge

"BioMed Central will be the most significant development for disseminating the results of biomedical research in our lifetime. "

Sir Paul Nurse, Cancer Research UK

Your research papers will be:

- available free of charge to the entire biomedical community

- peer reviewed and published immediately upon acceptance

- cited in PubMed and archived on PubMed Central

- yours - you keep the copyright 